\title{
Orbital Disordering and metal-insulator transition with hole-doping in perovskite-type vanadium oxides
}

\author{
J. Fujioka ${ }^{1}$, S. Miyasaka ${ }^{1}$, and Y. Tokura ${ }^{1,2,3}$ \\ ${ }^{1}$ Department of Applied Physics, University of Tokyo, Tokyo 113-8656, Japan \\ ${ }^{2}$ Spin Superstructure Project, ERATO, \\ Japan Science and Technology Agency, Tsukuba, 305-8562, Japan and \\ ${ }^{3}$ Correlated Electron Research Center (CERC), \\ National Institute of Advanced Industrial Science \\ and Technology (AIST), Tsukuba 305-8562, Japan
}

(Dated: September 12, 2018)

\begin{abstract}
Filling-control metal-insulator transitions (MITs) and related electronic phase diagrams have been investigated for hole-doped vanadium oxides, $\operatorname{Pr}_{1-x} \mathrm{Ca}_{x} \mathrm{VO}_{3}, \mathrm{Nd}_{1-x} \mathrm{Sr}_{x} \mathrm{VO}_{3}$ and $\mathrm{Y}_{1-x} \mathrm{Ca}_{x} \mathrm{VO}_{3}$, with perovskite structure. The increase of the doping level $x$ causes the melting of the $G$-type (and $C$-type) orbital order, prior to or concomitantly with the MIT, due partly to the doped-hole motion and partly to the ramdom potential arising from the quenched disorder. In particular, the $G$-type spin- and $C$-type orbital-ordered phase present in $\mathrm{Y}_{1-x} \mathrm{Ca}_{x} \mathrm{VO}_{3}$ disappears immediately upon hole doping, around $x=0.02$. On the other hand, the critical doping level $x$ for MIT is governed by the electron-correlation strength of the undoped parent compound.
\end{abstract}

PACS numbers: 71.30.+h, 71.27.+a, 75.30.Kz 
The orbital degree of freedom in $3 d$ transition-metal compounds has been attracting much attention, since it plays an important role not only in producing versatile magnetic structures but also in dramatically modefying charge transport as observed in the metal-insulator transition (MIT) and the colossal magnetoresistance phenomena [1, 2]. In particular, recent experimental and theoretical studies on perovskite-type manganites have clarified the close interplay among orbital, spin, charge and lattice degrees of freedom [3]. In manganese oxides the strong Jahn-Teller effect of the partially filled $e_{g}$-orbital dominates the lattice-coupled charge dynamics, as manifested by, for example, the charge/orbital ordering and disordering accompanying the metal-insulator phenomena. In the $t_{2 g}$-orbital sector, by contrast, the orbital-lattice interaction is much weaker than that in the $e_{g}$ ones. Therefore, the interplay between orbitals and spins, not only the intersite exchange interaction but also the intraatomic spin-orbit interaction, is more visible in vanadium oxides with active $t_{2 g}$-orbital electrons [4, 5].

A recent investigation on $\mathrm{La}_{1-x} \mathrm{Sr}_{x} \mathrm{VO}_{3}$ has revealed the critical role of the $t_{2 g}$ orbital and spin correlations near the boundary of the MIT [6]. The parent compound $\mathrm{LaVO}_{3}$ with perovskite structure is a prototypical Mott-Hubbard insulator, where $\mathrm{V}$ is nominally trivalent and has $3 d^{2}$ configuration, that is, two valence electrons in the $3 d$ orbitals $\left(t_{2 g}\right.$ manifold) with spin $\mathrm{S}=1$. Since the orthorhombic distortion lifts the degeneracy of the energy level of $t_{2 g}$ orbitals, one electron always occupies the $d_{x y}$ orbital and the other one either $d_{y z}$ or $d_{z x}$ orbital. With lowering temperature $(T), \mathrm{LaVO}_{3}$ undergoes two successive phase transitions [7, 8]. First, the magnetic transition from paramagnetic (PM) to $C$-type spin ordered state, where spins align ferromagnetically along the $c$ axis and stagger in the $a b$ plane, occurs at $T_{\mathrm{SO} 1}=143 \mathrm{~K}$. Subsequently, the structural phase transition accompanying the $G$ type orbital ordering $(\mathrm{OO})$, where $d_{y z}$ and/or $d_{z x}$ orbitals stagger in all $(x, y, z)$ directions, occurs at $T_{\mathrm{OO} 1}=141 \mathrm{~K}$ as shown Fig.1(a) [4]. By partially replacing La with $\mathrm{Sr}$, which results in hole doping (or decreasing band-filling), the filling-control MIT can be achieved. The sequential order and causality of the $C$-type spin ordering (SO) and the $G$-type OO are also observed in $\mathrm{La}_{1-x} \mathrm{Sr}_{x} \mathrm{VO}_{3}$ in an insulating region $(x<0.178)$. The MIT occurs around $x=0.178$ for $\mathrm{La}_{1-x} \mathrm{Sr}_{x} \mathrm{VO}_{3}$ accompanying the melting of the $G$-type $\mathrm{OO}$ and related structural phase transition, while the $C$-type SO remains up to around $x=0.260$ and forms the antiferromagnetic metallic state.

When La is replaced with other rare-earth elements $(R)$ and accordingly the 
orthorhombic-lattice distortion of the perovskite structure is changed, many of $R \mathrm{VO}_{3}$ show different $T$-dependent sequential order of the $\mathrm{SO}$ and $\mathrm{OO}$ transitions [4]. Additionally, some of them bear the different pattern of SO and OO, i.e. the $G$-type SO and $C$-type OO as the ground state. The whole spin-orbital phase diagram of $R \mathrm{VO}_{3}[4]$ is reproduced in Fig.1(a). In $R \mathrm{VO}_{3}$ with $R=\operatorname{Pr}$ to $\mathrm{Lu}$, the $G$-type $\mathrm{OO}$ appears well above $T_{\mathrm{SO} 1}$ and accordingly the $G$ type orbital-ordered but PM phase exists in the phase diagram. Thus, the sequential order and causality concerning the $C$-type $\mathrm{SO}$ and the $G$-type $\mathrm{OO}$ are opposite between $R \mathrm{VO}_{3}$ with $R=\operatorname{Pr}$ to $\mathrm{Lu}$ and those with $R=\mathrm{La}$ and Ce. As the typical examples of $R \mathrm{VO}_{3}$ showing the $\mathrm{OO}$ first, $\mathrm{PrVO}_{3}$ and $\mathrm{NdVO}_{3}$ are to be investigated here. In $R \mathrm{VO}_{3}$ with $R=$ Dy to $\mathrm{Lu}$, on the other hand, the $G$-type SO and $C$-type OO appear in the low- $T$ region (see Fig. 1(a)). Among them, the spin- and orbital-ordered state for $\mathrm{YVO}_{3}$ has been studied as the prototype experimentally [9, 10, 11, 12] and theoretically 13, 14]. As in $\mathrm{La}_{1-x} \mathrm{Sr}_{x} \mathrm{VO}_{3}$, the hole doping by partially replacing the trivalent $R$ with the divalent alkaline earth ones $(A=\operatorname{Sr}$ or $\mathrm{Ca}$ ), causes the MIT. We have chosen $\mathrm{Pr}_{1-x} \mathrm{Ca}_{x} \mathrm{VO}_{3}, \mathrm{Nd}_{1-x} \mathrm{Sr}_{x} \mathrm{VO}_{3}$ and $\mathrm{Y}_{1-x} \mathrm{Ca}_{x} \mathrm{VO}_{3}$ as the hole doped systems for $\mathrm{PrVO}_{3}, \mathrm{NdVO}_{3}$ and $\mathrm{YVO}_{3}$, respectively. To clarify the behavior of $\mathrm{SO}$ and $\mathrm{OO}$ in the hole doped region and the critical behavior of $\mathrm{OO}$ in the vicinity of MIT for these compounds, we have prepared the single crystals by a floating zone method [6] and systematically investigated transport, specific heat and magnetization with varying $x$.

The $T$-dependence of the resistivity $\rho$ for $\operatorname{Pr}_{1-x} \mathrm{Ca}_{x} \mathrm{VO}_{3}$ is shown in Fig. 2. The $\rho$ for $0 \leq x \leq 0.23$ shows an insulating behavior and the extrapolated zero- $T$ conductivity remains zero, while finite for $x>0.25$. This indicates that the filling-control MIT at zero- $T$ seems to occur at $x \sim 0.25$. The critical doping level $x_{c}$ for the MIT is similarly determined for $\mathrm{Nd}_{1-x} \mathrm{Sr}_{x} \mathrm{VO}_{3}\left(x_{c} \sim 0.23\right)$. The $x_{c}(=0.5)$ for $\mathrm{Y}_{1-x} \mathrm{Ca}_{x} \mathrm{VO}_{3}$ was reported in ref.[15]. In general, the partial substitution of $R$ with $A$ changes not only the nominal hole concentration but also the change of crystal structure and accordingly leads to the change of the effective oneelectron bandwidth. To see how the change of the effective one-electron bandwidth affects the MIT, we plotted the tolerance factor of each compound as a function of the doping level as shown in Fig.1 (b). In this regime, the tolerance factor represents the relative oneelectron bandwidth[16]. As seen in Fig. 1(b), $x_{c}$ increases systematically with decreasing the tolerance factor, or equivalently with decreasing the effective one-electron bandwidth as observed for $R_{1-x} A_{x} \mathrm{TiO}_{3}$ 17]. This is also consistent with the theoretical results [1, 18].

The $T$-dependence of the specific heat and magnetization for $\mathrm{Y}_{1-x} \mathrm{Ca}_{x} \mathrm{VO}_{3}, \mathrm{Nd}_{1-x} \mathrm{Sr}_{x} \mathrm{VO}_{3}$, 
and $\mathrm{Pr}_{1-x} \mathrm{Ca}_{x} \mathrm{VO}_{3}$ is shown in Fig. 3. In the respective end $(x=0)$ compounds, specific heat curves show two or three peaks corresponding to magnetic transitions and/or structural ones coupled with $\mathrm{OO}$, as previously reported [4]. The peaks at $T_{\mathrm{OO} 1}$ and $T_{\mathrm{SO} 1}$ correspond to the onset of the $G$-type $\mathrm{OO}$ and the $C$-type SO, respectively. The specific-heat jump observed for $\mathrm{YVO}_{3}$ at $T_{\mathrm{SO} 2}\left(=T_{\mathrm{OO} 2}\right)$ corresponds to the first-order transition to the $G$-type spin- and the $C$-type orbital-ordered phase. The magnetization curve also shows upturn at $T_{\mathrm{SO} 1}$ and jump at $T_{\mathrm{SO} 2}$, while no anomaly is observed at $T_{\mathrm{OO} 1}$. Also in the $\mathrm{Ca}$ - or $\mathrm{Sr}$ doped compounds the specific heat and magnetization curves show anomalies corresponding to the appearance of $\mathrm{SO}$ and $\mathrm{OO}$. At first, we focus on $\mathrm{Y}_{1-x} \mathrm{Ca}_{x} \mathrm{VO}_{3}$ among these three compounds. For $x=0.01$, the specific heat curve shows three discontinuities as seen in the end compound $(x=0)$. For $x=0.02$, however, the anomalies corresponding to the transition to the $G$-type spin- and $C$-type orbital-ordered phase cannot be observed down to $2 \mathrm{~K}$, while the other two peaks can be clearly as well. This indicates that the $G$-type spin- and $C$-type orbital-ordered phase is fragile against the hole doping and disappears at such a low doping level as $x=0.02$. With increasing $x$, the peak corresponding to the onset of the $G$-type OO gradually broadens, shifts to lower $T$, and above $x=0.10$ is hardly discerned. Thus, the correlation of the $G$-type OO becomes weaker with increasing $x$ and the $G$-type OO finally melts around $x=0.10$. With increasing $x$, the peak corresponding to the transition to the $C$-type SO also gradually broadens and consequently no peak can be discerned for $x=0.11$. The magnetization curve, however, shows upturn around $100 \mathrm{~K}$, indicating that the $C$-type SO still exists in this compound as shown in Fig. 3(d). This upturn can be seen up to $x \sim 0.6$, and consequently the $C$-type $\mathrm{SO}$ seems to exist even in a metallic region as in the case of $\mathrm{La}_{1-x} \mathrm{Sr}_{x} \mathrm{VO}_{3}$. These doping-dependent features are summarized in Fig. 4 as the electronic phase diagram.

As shown in Figs. 3(b), (c), (e) and (f), the specific heat curves for $\mathrm{Nd}_{1-x} \mathrm{Sr}_{x} \mathrm{VO}_{3}$ and $\mathrm{Pr}_{1-x} \mathrm{Ca}_{x} \mathrm{VO}_{3}$ show similar behaviors to those for $\mathrm{Y}_{1-x} \mathrm{Ca}_{x} \mathrm{VO}_{3}$ apart from the difference in the ground state at $x=0$. For $\mathrm{Nd}_{1-x} \mathrm{Sr}_{x} \mathrm{VO}_{3}$ and $\mathrm{Pr}_{1-x} \mathrm{Ca}_{x} \mathrm{VO}_{3}$, the peak corresponding to the onset of the $G$-type $\mathrm{OO}$ at $T_{\mathrm{OO} 1}$ disappears around $x=0.10$ and $x=0.20$, respectively, indicating the melting of the $G$-type OO. It is noted that the peak for $\operatorname{Pr}_{1-x} \mathrm{Ca}_{x} \mathrm{VO}_{3}$ can be observed up to a larger $x$ value than those for $\mathrm{Y}_{1-x} \mathrm{Ca}_{x} \mathrm{VO}_{3}$ and $\mathrm{Nd}_{1-x} \mathrm{Sr}_{x} \mathrm{VO}_{3}$, indicating that the long-range $G$-type $\mathrm{OO}$ remains up to a higher doped region for $\mathrm{Pr}_{1-x} \mathrm{Ca}_{x} \mathrm{VO}_{3}$. The magnetization curve also shows upturn corresponding to the onset of the $C$-type $\mathrm{SO}$ at $T_{\mathrm{SO} 1}$ 
up to $x=0.26$ for $\mathrm{Nd}_{1-x} \mathrm{Sr}_{x} \mathrm{VO}_{3}$ and up to $x=0.30$ for $\mathrm{Pr}_{1-x} \mathrm{Ca}_{x} \mathrm{VO}_{3}$ (see Fig.4), respectively.

The $T$-dependence of $\rho$ in a small $x$ region for $\operatorname{Pr}_{1-x} \mathrm{Ca}_{x} \mathrm{VO}_{3}$ also shows broad kinks which perhaps reflect the transition to the $G$-type $\mathrm{OO}$ and the $C$-type $\mathrm{SO}$ as shown in Fig. 2 . This contrasts with the clear kink due to the first-order phase transition observed in $\mathrm{La}_{1-x} \mathrm{Sr}_{x} \mathrm{VO}_{3}$ 6]. To see the anomaly more clearly, the $T$-derivative of logarithmic $\rho(d \log \rho / d T)$ is shown in the inset of Fig. 2. For $x=0.10$, it shows two dips around $T_{\mathrm{OO} 1}$ and $T_{\mathrm{SO} 1}$. For $x=0.25$, only the broad dip corresponding to the onset of the $C$-type $\mathrm{SO}$ can be seen around $T_{\mathrm{SO} 1}$. The evolution of the $C$-type SO seems to supress the charge transport effectively, since $d \log \rho / d T$ increases steeply below $T_{\mathrm{SO} 1}$ for the both compounds .

The electronic phase diagram, obtained by plotting the transition temperatures of the $\mathrm{SO}$ and $\mathrm{OO}$ as a function of $x$, is shown for each compound in Fig. 4, which includes that of $\mathrm{La}_{1-x} \mathrm{Sr}_{x} \mathrm{VO}_{3}$ previously reported [6]. For $\mathrm{Pr}_{1-x} \mathrm{Ca}_{x} \mathrm{VO}_{3}, \mathrm{Nd}_{1-x} \mathrm{Sr}_{x} \mathrm{VO}_{3}$ and $\mathrm{Y}_{1-x} \mathrm{Ca}_{x} \mathrm{VO}_{3}$, $T_{\mathrm{OO} 1}$ and $T_{\mathrm{SO} 1}$ systematically decrease with increasing $x$ and the $T$ interval between them also decreases for these three compounds. As mentioned above, the $G$-type OO seems to melt or at least become obscure around $x=0.10$ for $\mathrm{Y}_{1-x} \mathrm{Ca}_{x} \mathrm{VO}_{3}, x=0.09$ for $\mathrm{Nd}_{1-x} \mathrm{Sr}_{x} \mathrm{VO}_{3}$ and $x=0.20$ for $\operatorname{Pr}_{1-x} \mathrm{Ca}_{x} \mathrm{VO}_{3}$, respectively. Thus, the doping level where the $G$-type OO melts (defined as $x_{\mathrm{o}}$ here) is smaller than $x_{c}$ for the MIT. This also contrasts with that the $G$-type $\mathrm{OO}$ remains until the MIT, i.e. $x_{o}=x_{c}$, in $\mathrm{La}_{1-x} \mathrm{Sr}_{x} \mathrm{VO}_{3}$ with $T_{\mathrm{SO} 1}$ locating always above $T_{\mathrm{OO} 1}$. If the hole motion alone could cause the collapse of the $G$-type OO, $x_{\mathrm{o}}$ would decrease with the increase of the effective one-electron bandwidth, i.e. the kinetic energy of the doped holes. However, $x_{\mathrm{o}}$ does not change systematically with the tolerance factor, or equivalently the effective one-electron bandwidth, in contrast to the case of $x_{c}$ for the MIT. Thus, there seems to be another factor which destabilizes the PM and G-type orbital-ordered phase in addition to the increase of the kinetic energy of holes. The most plausible one is the quenched disorder arising from the solid solution of $R$ (small) and $A$ (large) ions, as recently demonstrated by the investigation on manganese oxides 19, 20]. To see the effect of the quenched disorder it is reasonable to compare $\mathrm{Nd}_{1-x} \mathrm{Sr}_{x} \mathrm{VO}_{3}$ with $\operatorname{Pr}_{1-x} \mathrm{Ca}_{x} \mathrm{VO}_{3}$. These systems share the close value of the one-electron bandwidth in the doping level of $x \sim 0.2$, where the melting of the $G$-type OO is observed (see Fig.1(b)). We defined the variance of the $R / A$ ionic radii as a function of $x, \sigma^{2}=\Sigma\left(x_{i} r_{i}^{2}-r_{A}^{2}\right)$, as the measure of the magnitude of the quenched disorder [19]. Here, $x_{i}, r_{i}$ and $r_{A}$ are the fractional occupancies, the effective ionic radii of cations of $R$ and $A$, and the averaged ionic radius 
$\left(r_{A}=(1-x) r_{\mathrm{R}^{3+}}+x r_{\mathrm{A}^{2+}}\right)$, respectively. At $x=0.10, \sigma^{2}$ for $\mathrm{Nd}_{1-x} \mathrm{Sr}_{x} \mathrm{VO}_{3}$ is about $2.6 \times 10^{-5}$ $\mathrm{nm}^{2}$, whereas that for $\operatorname{Pr}_{1-x} \mathrm{Ca}_{x} \mathrm{VO}_{3} 3.2 \times 10^{-6} \mathrm{~nm}^{2}$ is one order of magnitude smaller. Since the effective one-electron bandwidth for $\mathrm{Nd}_{1-x} \mathrm{Sr}_{x} \mathrm{VO}_{3}$ is close to that for $\operatorname{Pr}_{1-x} \mathrm{Ca}_{x} \mathrm{VO}_{3}$ in this doping level, this suggests that as well as the motion of the doped hole the increase of the magnitude of the quenched disorder results in the disappearance of the long-range $G$ type OO. This is perhaps because the disorder randomizes the local lattice distortion which would be induced by the collective Jahn-Teller coupling distortion concomitant with the $G$ type OO, and hence plays a role of random field acting on the orbital (pseudo-spin) sector. To see the lattice distortion coupled with the G-type OO, Raman-scattering spectroscopy can be used because of its sensitivity to the lattice distortion. For $\mathrm{NdVO}_{3}$, an activated Raman mode is observed at around $700 \mathrm{~cm}^{-1}$ below $T_{\mathrm{OO} 1}[4]$. With increasing $x$ the peak broadens and its integrated intensity also decreases. This is indicative of the suppression of the lattice distortion coupled with the $G$-type OO. At $x=0.12$ for $\mathrm{Nd}_{1-x} \mathrm{Sr}_{x} \mathrm{VO}_{3}$ where the long-range PM and G-type orbital-ordered phase seems to be absent, a broad peak-structure can be observed in a low- $T$ region, suggesting the subsistence of the short range correlation of the $G$-type OO. Quite a similar disorder-induced melting of the long range OO into the short-range correlation has also been reported for manganites[21]. On the other hand, the $C$-type SO is robust against the increase of $x$ and remains even in the metallic region, which is similar to the case of $\mathrm{La}_{1-x} \mathrm{Sr}_{x} \mathrm{VO}_{3}$. The effect of the quenched disorder is much less on the $C$-type SO than on the $G$-type OO, perhaps reflecting the smaller coupling of SO with the lattice. Finally, some remarks should be added about the doping effect on the $G$-type spin- and the $C$-type orbital-ordered phase in $\mathrm{Y}_{1-x} \mathrm{Ca}_{x} \mathrm{VO}_{3}$. As observed in Fig. 4, the $G$-type spin- and $C$-type orbital-ordered phase is extremely unstable against such a small hole doping as $x=0.02$. The magnitude of the quenched disorder is supposed to be minimal in such a lightly doped region. A recent theoretical calculation[22] predicts that the motion of the doped hole reduces the spin order parameter, which causes the softening of orbiton and leads to the instability of the $G$-type spin- and the $C$-type orbital-ordered phase. The extreme sensitivity to the doping level may also arise from the bicritical phase competition with the adjacent $G$-type $\mathrm{OO}$ and $C$-type $\mathrm{SO}$ phase that governs the ground state phase for $0.02<x<0.10$.

In summary, we have revealed the electronic phase diagram for $\operatorname{Pr}_{1-x} \mathrm{Ca}_{x} \mathrm{VO}_{3}$, $\mathrm{Nd}_{1-x} \mathrm{Sr}_{x} \mathrm{VO}_{3}$ and $\mathrm{Y}_{1-x} \mathrm{Ca}_{x} \mathrm{VO}_{3}$ by the transport, specific heat and magnetization measure- 
ments. The paramagnetic and long-range $G$-type orbital ordered phase is unstable against the increase of the doping level $x$, which is attributed not only to the hole motion but also to the increase of the quenched disorder arising from the random chemical substitution of the rare-earth elements with alkaline earth ones. On the other hand, the critical doping level for the insulator-metal transition is governed by the one-electron bandwidth. Moreover, the $G$-type spin- and the $C$-type orbital-ordered phase for $\mathrm{Y}_{1-x} \mathrm{Ca}_{x} \mathrm{VO}_{3}$ disappears at a minimal doping as low as $x=0.02$ and, hence, is extremely sensitive to the change of band-filling, as compared with the $C$-type spin- and $G$-type orbital-ordered one.

We would thank S. Ishihara for helpful discussion.

[1] For a review, M. Imada, A. Fujimori and Y. Tokura, Rev. Mod. Phys. 70, 1039 (1998).

[2] Y. Tokura and N. Nagaosa, Science 288, 462 (2000).

[3] Colossal Magnetoresistive Oxides, vol. 2 of Advances in Condensed Matter Science edited by Y. Tokura (Gordon and Breach, Amsterdam, 2000).

[4] S. Miyasaka, et al., Phys. Rev. B 68, 100406(R) (2003).

[5] Y. Motome, H. Seo, Z. Fang and N. Nagaosa, Phys. Rev. Lett. 90, 146602 (2003).

[6] S. Miyasaka, T. Okuda, and Y. Tokura, Phys. Rev. Lett. 85, 5388 (2000).

[7] V.G. Zubkov, et al., Sov, Phys. Solid State, 18,1165 (1976).

[8] P. Bordet, et al., J.Solid State Chem, 106, 253(1993).

[9] G.R.Blake et al., Phys. Rev. B. 65, 174112 (2002).

[10] M. Noguchi et al., Phys. Rev. B. 62, R9271 (2000).

[11] C. Ulrich et al., Phys. Rev. Lett. 91, 257202 (2003).

[12] H. Kawano et al., J. Phys. Soc. Jpn, 63, 2857(1994).

[13] G. Khaliullin, P. Horsch and A. Oles, Phys. Rev. Lett. 86, 3879 (2001).

[14] H. Sawada, et al., Phys. Rev. B, 53, 12742(1996).

[15] M. Kasuya et al., Phys. Rev. B. 47, 6197 (1993).

[16] Y.Okimoto et al., Phys. Rev. B. 51, 9581(1995)

[17] T. Katsufuji, Y. Taguchi and Y. Tokura, Phys. Rev. B. 56, 10145 (1997).

[18] M.Imada, J. Phys. Soc. Jpn. 64, 2954 (1995).

[19] L. M. Rodriguez-Martinez and J. P. Attfield, Phys. Rev. B. 63, 024424 (2000). 
[20] Y. Tomioka and Y. Tokura, Phys. Rev. B. 70, 014432 (2004).

[21] Y. Tomioka et al., Phys. Rev. B. 68, 094417 (2003).

[22] S. Ishihara, cond-mat/040839 v1 18 Aug 2004. 
FIG. 1: (a) The spin-orbital phase diagram in $R \mathrm{VO}_{3}(R=\mathrm{Lu}-\mathrm{La})$. Closed and open circles, and open triangle indicate the transition temperatures of the $G$-type orbital ordering $(\mathrm{OO}), T_{\mathrm{OO} 1}$, the $C$-type spin ordering $(\mathrm{SO}), T_{\mathrm{SO} 1}$, and the $G$-type $\mathrm{SO}$ and the $C$-type $\mathrm{OO}, T_{\mathrm{SO} 2}=T_{\mathrm{OO} 2}$, respectively. Schematic representations of the $G$-type OO, the $G$-type SO and $C$-type OO, and the $C$-type $\mathrm{OO}$ and $G$-type $\mathrm{OO}$ are also shown, in which open arrows and lobes indicate spins, and occupied $d_{y z}$ and $d_{z x}$ orbitals on the vanadium ions, respectively. (b) The doping level versus the tolerance factor for $\mathrm{La}_{1-x} \mathrm{Sr}_{x} \mathrm{VO}_{3}, \mathrm{Pr}_{1-x} \mathrm{Ca}_{x} \mathrm{VO}_{3}, \mathrm{Nd}_{1-x} \mathrm{Sr}_{x} \mathrm{VO}_{3}$ and $\mathrm{Y}_{1-x} \mathrm{Ca}_{x} \mathrm{VO}_{3}$. Open circles indicates the critical doping level $x_{c}$ of the metal-insulator transition.

FIG. 2: Temperature dependence of resistivity for single crystals of $\operatorname{Pr}_{1-x} \mathrm{Ca}_{x} \mathrm{VO}_{3}$ with various $x$. The inset shows the temperature derivative of logarithmic resistivity $(d \log \rho / d \mathrm{~T})$. The closed and open triangles indicate the transition temperatures of the $G$-type $\mathrm{OO}$ and the $C$-type SO, respectively.

FIG. 3: The temperature dependence of specific heat and magnetization for $\mathrm{Y}_{1-x} \mathrm{Ca}_{x} \mathrm{VO}_{3}((\mathrm{a}),(\mathrm{d}))$, $\mathrm{Nd}_{1-x} \mathrm{Sr}_{x} \mathrm{VO}_{3}((\mathrm{~b}),(\mathrm{e}))$, and $\mathrm{Pr}_{1-x} \mathrm{Ca}_{x} \mathrm{VO}_{3}((\mathrm{c}),(\mathrm{f}))$. The closed, open and double triangles indicate the transition temperatures of the $G$-type OO, the $C$-type SO, and the $G$-type $\mathrm{SO}$ and $C$-type OO, respectively.

FIG. 4: The electronic phase diagram as a function of the doping level $x$ for $\mathrm{La}_{1-x} \mathrm{Sr}_{x} \mathrm{VO}_{3}[\underline{6}]$, $\mathrm{Pr}_{1-x} \mathrm{Ca}_{x} \mathrm{VO}_{3}, \mathrm{Nd}_{1-x} \mathrm{Sr}_{x} \mathrm{VO}_{3}$ and $\mathrm{Y}_{1-x} \mathrm{Ca}_{x} \mathrm{VO}_{3}$. The open, closed circles, and open triangles indicate the transition temperatures of the $G$-type $\mathrm{OO}\left(T_{\mathrm{OO} 1}\right)$, the $C$-type $\mathrm{SO}\left(T_{\mathrm{SO} 1}\right)$ and the $G$-type $\mathrm{SO}$ and the $C$-typeOO $\left(T_{\mathrm{OO} 2}=T_{\mathrm{SO} 2}\right)$, respectively. 
This figure "fig1.JPG" is available in "JPG" format from: http://arxiv.org/ps/cond-mat/0505190v1 
This figure "fig2.JPG" is available in "JPG" format from: http://arxiv.org/ps/cond-mat/0505190v1 
This figure "fig3.JPG" is available in "JPG" format from: http://arxiv.org/ps/cond-mat/0505190v1 
This figure "fig4.JPG" is available in "JPG" format from: http://arxiv.org/ps/cond-mat/0505190v1 